\title{
The Hausdorff Nearest Circle to a Triangle
}

\author{
I. Ginchev
}

\begin{abstract}
The problem of finding the nearest in the Hausdorff metrics circle to a non-empty convex compact set $T$ in the plane is considered. It is shown that this problem is equivalent to the problem of the Chebyshevian best approximation of $2 \pi$-periodic functions by trigonometric polynomials of first order and in consequence the Hausdorff nearest circle is unique. The case when $T$ is a triangle is solved completely. It is shown then that the center of the nearest circle is the intersection point of the midline of the longest side and the bisectrix against the shortest side of the triangle.
\end{abstract}

Keywords: Convex sets in two dimensions, Hausdorff metrics, approximation by circles AMS subject classification: $52 \mathrm{~A} 10,52 \mathrm{~A} 27$

\section{Preliminaries}

The following problem comes from the praxis: A machine tool $T$ is supposed to be a circle (ideal circle) but its true form (non-ideal circle) declines from the ideal one within admitted limits. A natural question arises, if this tool is recognized as a circle: what is the radius it has to be given (and how it can be measured)? Sometimes the practical solution of this problem is the following one: The polar equation $r=r(\theta)$ of $T$ related to a point $O \in T$ is written (provided $T$ is star-like with respect to $O$ ) and then the mean value $R=\frac{1}{2 \pi} \int_{0}^{2 \pi} r(\theta) d \theta$ is the radius to be given to $T$ (often the arithmetic mean: of a finite sum is taken instead of the integral). Obviously, from mathematical point of view such an approach is not acceptable, since the value $R$ obtained in this way depends on the choice of $O$.

We accept as natural the following procedure. Let $\mathcal{K}$ be the set of all circles on the plane and $h(A, B)$ be a certain functional measuring the distance between sets $A$ and $B$. Provided there is a unique circle $\hat{K}=K(\hat{O}, \hat{\rho})$, being a solution of the problem

$$
h(T, K) \rightarrow \min \quad(K \in \mathcal{K})
$$

its radius $\hat{\rho}$ is taken as the radius and its center $\hat{O}$ as the center to be given to $T$. Still there can be a discussion, which can be the most appropriate distance measure $h$. The approach depends on the purpose and on the convenience. We take here $h$ to be the Hausdorff distance, which is appropriate to the spirit of the practical problem. As a simplification to the real situation we take $T$ to be a non-empty convex compact set (if this is not the case, then we deal with the convex hull of $T$ ).

I. Ginchev: Technical University, Department of Mathematics, BG - 9010 Varna, Bulgaria 
We use the following notations:

$\mathbb{E}$ is the Euclidean plane.

$\vec{p} \cdot \vec{q}$ is the scalar product of two vectors $\vec{p}$ and $\vec{q}$ in $\mathbb{E}$.

$\mathcal{T}$ is the set of all non-empty convex compact sets on $\mathbb{E}$.

$\mathcal{K}$ is the set of all the circles (two-dimensional balls) on $\mathbb{E}$.

$\mathcal{K}(X)$ is the set of all the circles in $\mathbb{E}$ with fixed centers at $X$.

$B$ is the unit ball in $E$.

$S$ is the unit circumference in $\mathbb{E}$.

$h\left(T_{1}, T_{2}\right)$ is the Hausdorf distance between sets $T_{1}, T_{2} \in \mathcal{T}$.

$s_{T}$ is the support function of a set $T \in \mathcal{T}$.

Let us mention that we distinguish between (free) vectors (written with vector accents) and points in $\mathbb{E}$. Obviously, $\mathcal{K}(X) \subset \mathcal{K} \subset \mathcal{T}$. Remind that

$$
h\left(T_{1}, T_{2}\right)=\inf \left\{\epsilon>0 \mid T_{1} \subset T_{2}+\epsilon B \text { and } T_{2} \subset T_{1}+\epsilon B\right\} .
$$

The support function $s_{T}$ is related to a given original point $O$; in order to underline this we write $s_{T, O}$ instead of $s_{T}$. The arguments of $s_{T}$ are vectors on $\mathbb{E}$. Remind that

$$
s_{T}(\vec{e})=\max _{M \in T} \vec{e} \cdot \overrightarrow{O M} \text {. }
$$

Obviously, if $O$ and $X$ are two points in $E$, then

$$
s_{T, O}(\vec{e})=\vec{e} \cdot \overrightarrow{O X}+s_{T, X}(\vec{e}) .
$$

Further we consider $s_{T}$ as a function on $S$. It is known (see, e.g., Leichtweiß [10]) that

$$
h\left(T_{1}, T_{2}\right)=\left\|s_{T_{1}}-s_{T_{2}}\right\| s:=\max _{\vec{e} \in S}\left|s_{T_{1}}(\vec{e})-s_{T_{2}}(\vec{e})\right|
$$

where $\|\cdot\|_{S}$ stands for the Chebyshevian norm on $S$. In (2) both $s_{T_{1}}$ and $s_{T_{2}}$ are related to the same original point. Thus problem (1) has the following analytic setting:

$$
h(T, K)=\left\|s_{T}-s_{K}\right\|_{S} \longrightarrow \min \quad(K \in \mathcal{K}) .
$$

Let $K_{0}$ be a fixed non-degenerate circle and let $\mathcal{G}$ be the group consisting of the translations and the homotheties on the plane. Then we have the problem

$$
h(T, K)=\left\|s_{T}-s_{\kappa}\right\|_{S} \longrightarrow \min \quad\left(K \in \mathcal{G} K_{0}\right)
$$

Similar problem was considered by Arnold [2] taking as distance the $L^{2}$-norm

$$
h(T, K)=\left(\int_{S}\left|s_{T}(\vec{e})-s_{K}(\vec{e})\right|^{2} d o(\vec{e})\right)^{1 / 2}
$$


as $\mathcal{G}$ the translation group and as $K_{0}$ an arbitrary compact convex set. He proves that the solution of this problem is the Steiner point of $\mathcal{G}$. With this remark we want to underline the resemblance of the two problems, not to provide discussion, whether similar in some sense result is valid in our case.

Concluding this section, let us mention the works of several authors. Alt and Wagener [1] give a computational procedure to find the circle of the best approximation for a convex polygon, provided the metric $h$ into consideration is the area of the symmetric difference between $T_{1}$ and $T_{2}$. More works are devoted to the best approximation by polygons. Florian [5] discusses the best approximation by polygons related to the cited area metrics. The best Hausdorff approximation of convex bodies by polygons is studied by Kenderov [8]. He proves certain equioscillation property. Various problems can be related to the problem of the best Hausdorff approximation of convex sets by circles. For instance, following Lessak [11] one proves easily that for each $T \in \mathcal{T}$ there exists an ellipse $K$ such that $h(T, K) \leq \frac{1}{4} \operatorname{diam} T$. The question is whether this inequality remains true putting for $K$ the Hausdorff best approximating circle of $T$. The affirmative answer of this question we intend to demonstrate in another publication. With consideration to the posed above practical problem, the main things remains for us to calculate numerically the Hausdorff best approximating circle. We see further that the problem leads to Chebyshevian approximation of continuous or more precisely of sinusoidal convex functions by trigonometric polynomials of first order. Well described algorithms for solving such problems one can find, e.g., in Brudnyj and Irodova [4] and Laurent [9] (Remez algorithm) or Hettich and Zenke [6] (linear programming). Some works, e.g. of Bani and Chalmers [3] suggest for a connection with the $L^{2}$ and $L^{p}$ norm. The geometric nature of the problem leads to certain simplifications, a matter that we are inclined to discuss in a future publication. Let us mention that the presented here case of a triangle already shows properties being characteristic for the general problem.

\section{The Hausdorff nearest circle to a convex compact set in the plane}

If $K=K(X, \rho)$ is a circle centered at $\mathrm{X}$ and having radius $\rho$, then $s_{K, X}=\rho$. Provided that the support function $s_{T}=s_{T, O}$ is related to a previously fixed original point $O$, we have the problem

$$
h(T, K)=\max _{\vec{e} \in S}\left\{\left|s_{T}(\vec{e})-(\rho+\vec{e} \cdot \overrightarrow{O X})\right|\right\} \rightarrow \min \quad(X \in E, \rho \geq 0) .
$$

Suppose that an orthogonal coordinate system originated at $O$ is introduced, and let $X=(a, b)$ and $\vec{e}=(\cos t, \sin t)$ be the coordinates of $X$ and $\vec{e}$, respectively. Then we come to the problem

$$
h(T, K)=\|s(t)-(\rho+a \cos t+b \sin t)\| \longrightarrow \min \quad(a, b \in \mathbb{R}, \rho \geq 0)
$$

where $s(t)=s_{T, O}(\cos t, \sin t)$ is a continuous (and sinusoidal convex) $2 \pi$-periodic function and $\|\cdot\|$ stands for the uniform norm on the interval $[0,2 \pi]$. 
So, our problem is equivalent to the problem of Chebyshevian approximation of a $2 \pi$-periodic continuous functions by trigonometric polynomials of first order. As it is well known (see, e.g., Karlin and Studden [7: Theorem 6.1]), this problem has a unique solution. Therefore we draw the following conclusion.

Theorem 1. For each non-empty convex compact set $T$ in the Euclidean plane $\mathbb{E}$ there exists a unique Hausdorff nearest circle.

The approximation problem (3) can be numerically solved applying the Remez or linear programming algorithm. Concerning here the casc of a triangle we will apply a direct treatment, splitting the problem of finding the Hausdorff nearest circle to a set $T \in \mathcal{T}$ into the following ones:

(P1) Find the Hausdorff nearest circle $\hat{K}_{X}$ to a set $T \in \mathcal{T}$ in the class $\mathcal{K}(X)$ of all circles with a fixed center $X$.

(P2) Find the Hausdorff nearest circle to a set $T \in \mathcal{T}$ among the circles $\hat{K}_{X}$ already obtained in problem (P1).

\section{The support function for a triangle}

Let $T=A_{1} A_{2} A_{3}$ be a triangle with vertices $A_{1}, A_{2}$ and $A_{3}$, following for determination in this order in counter clock-wise direction. We shall find an explicite expression for the support function $s_{T}=s_{T, X}$ related to the point $X$. In our further considerations $X$ is the center of the circle $K=K(X, \rho)$ and therefore

$$
h(T, K)=\left\|s_{T}-\rho\right\| \text {. }
$$

We introduce the following notations (see Figure 1):

- $\vec{e}_{1}, \vec{e}_{2}$ and $\vec{e}_{3}$ are the outer unit normals to the sides $A_{2} A_{3}, A_{3} A_{1}$ and $A_{1} A_{2}$, respectively.

- $\vec{a}_{i}=\overrightarrow{X A_{i}}$ and $r_{i}=\left|\vec{a}_{i}\right|(i=1,2,3)$, where $|\cdot|$ stands for the length of vectors.

- $d_{1}=\vec{a}_{2} \cdot \vec{e}_{1}=\vec{a}_{3} \cdot \vec{e}_{1}, d_{2}=\vec{a}_{3} \cdot \vec{e}_{2}=\vec{a}_{1} \cdot \vec{e}_{2}$ and $d_{3}=\vec{a}_{1} \cdot \vec{e}_{3}=\vec{a}_{2} \cdot \vec{e}_{3}$ are the oriented distances of $X$ to the straight lines defined by the sides.

- $r=r(X)=\max \left\{r_{1}, r_{2}, r_{3}\right\}$ and $d=d(X)=\min \left\{d_{1}, d_{2}, d_{3}\right\}$.

- $a_{1}, a_{2}$ and $a_{3}$ are the lengths of the sides $A_{2} A_{3}, A_{3} A_{1}$ and $A_{1} A_{2}$, respectively, and $A_{i}$ denotes also the measure of the angle of the triangle, whose vertex is at $A_{i}(i=1,2,3)$.

For three unit vectors $\vec{u}_{1}, \vec{u}_{2}$ and $\vec{u}_{3}$ we write $\vec{u}_{1} \leq \vec{u}_{2} \leq \vec{u}_{3}$ if their end points follow in counter clock-wise direction in this order (the used here "inequalities" define not a usual order, but a "cyclic" one). Further we put

$$
S_{1}=\left\{\vec{e} \in S \mid \vec{e}_{2} \leq \vec{e} \leq \vec{e}_{3}\right\}, \quad S_{2}=\left\{\vec{e} \in S \mid \vec{e}_{3} \leq \vec{e} \leq \vec{e}_{1}\right\}, \quad S_{3}=\left\{\vec{e} \in S \mid \vec{e}_{1} \leq \vec{e} \leq \vec{e}_{2}\right\} .
$$

Now it is obvious (or in fact easy to be proved) that

$$
s_{T}(\vec{e})=\vec{a}_{i} \cdot \vec{e} \quad\left(\vec{e} \in S_{i} ; i=1,2,3\right) .
$$

is the support function of the triangle. 
Lemma 1. The relation

$$
\max _{1 \leq i \leq 3} \max _{e \in S_{i}} \vec{a}_{i} \cdot \vec{e}=r
$$

is true.

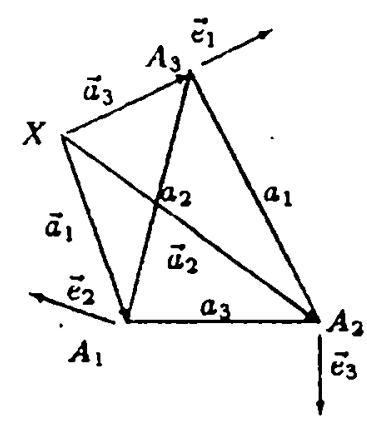

Figure 1

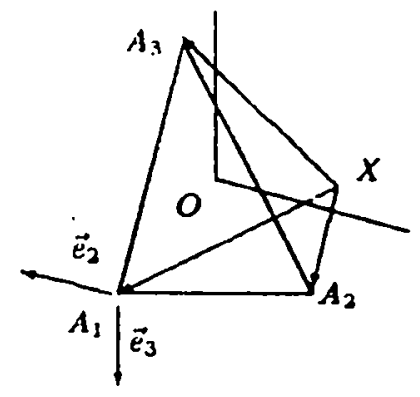

Figure 2

Proof. Put for brevity $\Delta_{i}=\max _{\vec{e} \in S_{i}} \vec{a}_{i} \cdot \vec{e}(i=1,2,3)$. Suppose for determination that $X$ lays in the angle formed by the midlines of the sides $A_{1} A_{2}$ and $A_{1} A_{3}$ (see Figure 2 ). With this remark it is clear that there exists a vector $\vec{e} \in S_{1}$ having the same direction as $\vec{a}_{1}$ and therefore $\Delta_{1}=\vec{a}_{1} \cdot \vec{e}=r_{1}$. Since obviously $\Delta_{i} \leq r_{i}$ we obtain (5)

We introduce the notation

$$
W_{i}=\left\{X \in E \mid \overrightarrow{A_{i} X} \cdot \overrightarrow{A_{i} Y} \leq 0 \text { for all } Y \in T\right\} \quad(i=1,2,3)
$$

Let $W$ be the closure of the completion $E \backslash\left(W_{1} \cup W_{2} \cup W_{3}\right)$ (for an illustration see Figure 5).

Lemma 2. It holds

$$
\min _{1 \leq i \leq 3} \min _{\vec{e} \in S_{i}} \vec{a}_{i} \cdot \dot{\vec{e}}=\left\{\begin{array}{ll}
d & \text { for } X \in W \\
-r_{i} & \text { for } X \in W_{i}
\end{array} \quad(i=1,2,3) .\right.
$$

Proof. Put $\delta_{i}=\min _{\vec{e} \in S_{i}} \vec{a}_{i} \cdot \vec{e}(i=1,2,3)$. Suppose for determination that the point $X$ is such that $d=d_{1}$ (see Figure 3 ). Then $\delta_{1}=\min \left\{\vec{a}_{1} \cdot \vec{e}_{2}, \vec{a}_{1} \cdot \vec{e}_{3}\right\}=\min \left\{d_{2}, d_{3}\right\}$. The following cases may arise:

Case $X \in W$. Then $\delta_{2}=\vec{a}_{2} \cdot \vec{e}_{1}=d_{1}, \delta_{3}=\vec{a}_{3} \cdot \vec{e}_{1}=d_{1}$ and $\min \left\{\delta_{1}, \delta_{2}, \delta_{3}\right\}=d_{1}$.

Case $X \in W_{2}$. Then $\delta_{2}=-r_{2}, \delta_{3}=\vec{a}_{3} \cdot \vec{e}_{2}=d_{1}$ and $\min \left\{\delta_{1}, \delta_{2}, \delta_{3}\right\}=\min \left\{d_{1}, d_{2}, d_{3} ;-r_{2}\right\}=-r_{2}$.

Case $X \in W_{3}$. Then like in the previous case

$$
\min \left\{\delta_{1}, \delta_{2}, \delta_{3}\right\}=\min \left\{d_{1}, d_{2}, d_{3},-r_{3}\right\}=-r_{3} \text {. }
$$

Thus the statement is proved 
Theorem 2. The Hausdorff distance between the triangle $T$ and the circle $K=$ $K(X, \rho)$ is given by

$$
h(T, K)= \begin{cases}\max \{r-\rho, \rho-d\} & \text { for } X \in W \\ \max \left\{r-\rho, \rho+r_{i}\right\} & \text { for } X \in W_{i}(i=1,2,3) .\end{cases}
$$

Proof. We have

$$
\begin{aligned}
h(T, K) & =\left\|s_{T, X}-\rho\right\| \\
& =\max _{\vec{e} \in S}\left|s_{T, X}(\vec{e})-\rho\right| \\
& =\max _{1 \leq i \leq 3} \max _{\vec{e} \in S_{i}} \max \left\{\vec{a}_{i} \cdot \vec{e}-\rho, \rho-\vec{a}_{i} \cdot \vec{e}\right\} \\
& \left.=\max \left\{\max _{1 \leq i \leq 3} \max _{\vec{e} \in S_{i}} \vec{a}_{i} \cdot \vec{e}-\rho, \rho-\min _{1 \leq i \leq 3} \min _{\vec{e} \in S_{i}} \vec{a}_{i} \cdot \vec{e}\right\}\right\}
\end{aligned}
$$

whence applying the previous two lemmas we obtain (6)

\section{Approximation with circles having fixed center}

Fix the point $X$. Let us suppose for a moment that $T \in T$ is arbitrary, i.e. not necessary a triangle. We want to find the Hausdorff nearest circle $K=K(X, \rho) \in \mathcal{K}(X)$. The radius $\rho$ is the solution of the problem $\left\|s_{T, X}-\rho\right\|_{S} \rightarrow \min (\rho \geq 0)$ which is convex and non-smooth in general. For a triangle $T$ this circle can be easily found.

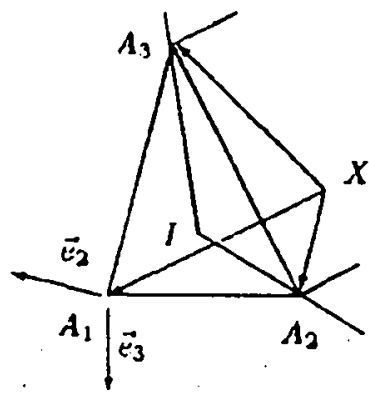

Figure 3

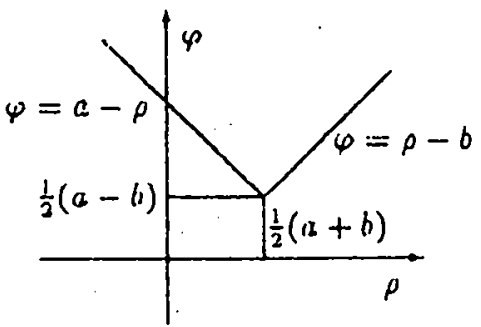

Figure 4

Using the notations from the previous section we have the following

Theorem 3. Among all the circles with a given center $X$ the best Hausdorff approximation to the triangle $T$ gives the circle, whose radius is

$$
\hat{\rho}(X)= \begin{cases}\frac{1}{2}(r+d) . & \text { for } X \in W . \\ \frac{1}{2}\left(r-r_{i}\right) & \text { for } X \in W_{i} \quad(i=1,2,3) .\end{cases}
$$


The corresponding Hausdorff distance is

$$
\hat{h}(X)= \begin{cases}\frac{1}{2}(r-d) & \text { for } X \in W \\ \frac{1}{2}\left(r+r_{i}\right) & \text { for } X \in W_{i} \quad(i=1,2,3) .\end{cases}
$$

Proof. For the function

$$
\varphi(\rho)=\max \{a-\rho, \rho-b\}
$$

it is true that

$$
\varphi_{\min }=\varphi\left(\frac{1}{2}(a+b)\right)=\frac{1}{2}(a-b) .
$$

An illustration is given on Figure 4. Now it remains to note that according to formula (6) the investigated distance is a function of type (7) with $a=r$ and either $b=d$ or $b=-r_{i}$ depending on whether $X \in W$ or $X \in W_{i}$

As a matter of fact this theorem solves problem (P1). Problem (P2) will be discussed in the next section.

\section{The Hausdorff nearest circle to a triangle}

In this section we solve Problem (P2) for a triangle.

Lemma 3. The function

$$
g(u, v)=\sqrt{a u^{2}+2 b u v+c v^{2}}+p u+q v+r \quad\left(a>0, a c-b^{2}>0\right)
$$

is convex. It possesses a local minimum if and only if

$$
a-p^{2} \geq 0 \quad \text { and } \quad a q^{2}-2 b p q+c p^{2} \leq a c-b^{2} .
$$

In this case the minimum $g_{\min }=r$ is attained for

$$
\begin{aligned}
& u=(c p-b q) t \\
& v=(a q-b p) t
\end{aligned} \quad \text { where } \begin{cases}t \leq 0 & \text { if } a q^{2}-2 b p q+c p^{2}=a c-b^{2} \\
t=0 & \text { if } a q^{2}-2 b p q+i p^{2}<a c-b^{2}\end{cases}
$$

Proof. Put for brevity

$$
Q=Q(u, v)=a u^{2}+2 b u v+c v^{2} \quad \text { and } \quad \Delta=a c-b^{2} .
$$

A differentiation gives

$$
\begin{gathered}
g_{u}=\frac{a u+b v}{\sqrt{Q}}+p, \quad g_{v}=\frac{b u+c v}{\sqrt{Q}}+q \\
g_{u u}=\frac{\Delta v^{2}}{\sqrt{Q^{3}}}, \quad g_{u v}=\frac{-\Delta u v}{\sqrt{Q^{3}}}, \quad g_{v v}=\frac{\Delta u^{2}}{\sqrt{Q^{3}}} .
\end{gathered}
$$


Since $g_{u u}>0$ and $g_{u v} g_{v v}-g_{u v}^{2}=0$, therefore $g(u, v)$ is a convex function. The critical points are the solutions of the system

$$
\begin{aligned}
& g_{u}(u, v)=0 \\
& g_{v}(u, v)=0
\end{aligned}
$$

We obtain the system

$$
\begin{aligned}
& a u+b v=-p \sqrt{Q} \\
& b u+c v=-q \sqrt{Q}
\end{aligned}
$$

which implies

$$
(a u+b v) q-(b u+c v) p=\dot{0} \Leftrightarrow(a q-b p) u+(b q-c p) v=0
$$

The following cases arise:

Case 1. $a q-b p=0$ and $b q-c p=0$. Since $\Delta \neq 0$, hence $p=q=0$ and $u=v=0$. In this case $a q^{2}-2 b p q+c p^{2}=0<a c-b^{2}$ and obviously

$$
g(u, v)=\sqrt{a u^{2}=2 b u v+c v^{2}}+r \geq r=g(0,0) .
$$

Case 2. $a q-b p \neq 0$ or $b q-c p \neq 0$, whence $p$ and $q$ are not both zero, say $p \neq 0$. We obtain

$$
u=(c p-b q) t \quad \text { and } \quad v=(a q-b p) t .
$$

The equation $a u+b v=-p \sqrt{Q}$ is transformed into

$$
\Delta t=-|t| \sqrt{\Delta\left(c p^{2}-2 b p q+a q^{2}\right)}
$$

which leads to the following two possibilities:

Subcase 2.1. $t=0$ whence $u=0$ and $v=0$. The curious point is that not every critical point of a convex function of two variables should give a minimum. For this reason we give additional arguments. In a neighbourhood of $(\hat{u}, \hat{v})=(0,0)$ we have

$$
\begin{aligned}
g(u, v)-r & =\sqrt{a u^{2}+2 b u v+c v^{2}}+p u+q u \\
& \geq \sqrt{a u^{2}+2 b u v+c v^{2}}-\sqrt{p^{2} u^{2}+2 p q u v+q^{2} v^{2}} \\
& \geq 0
\end{aligned}
$$

if

$$
a u^{2}+2 b u v+c v^{2} \geq p^{2} u^{2}+2 p q u v+q^{2} v^{2} \text { for all }(u, v) \text {. }
$$

The latter is equivalent to

$$
a-p^{2} \geq 0 \quad \text { and } \quad\left(a-p^{2}\right)\left(c-q^{2}\right)-(b-p q)^{2} \geq 0
$$


i.e. when (8) is true. If $a-p^{2}<0$ and $s<0$ we have $g(s, 0)=(p-\sqrt{a}) s+r<r=g(\hat{u}, \hat{v})$. Suppose that $a q^{2}-2 b p q+c p^{2}>a c-b^{2}$. Then for $s<0$ we have

$$
\begin{aligned}
& g(s(c p-b q), s(a q-b p)) \\
& \quad=s\left(-\sqrt{\Delta\left(c p^{2}-2 b p q+a q^{2}\right)}+c p^{2}-2 b p q+a q^{2}\right)+r \\
& \quad<s\left(-\Delta+c p^{2}-2 b p q+a q^{2}\right)+r \\
& \quad<r=g(\hat{u}, \hat{v}) .
\end{aligned}
$$

Subcase 2.2. $\sqrt{\Delta} \operatorname{sign} t=-\sqrt{\Delta\left(c p^{2}-2 b p q+a q^{2}\right)}$ whence we get the critical points

$$
\hat{u}=(c p-b q) t \quad \text { and } \quad \hat{v}=(a q-b p) t \quad(t \leq 0) .
$$

If also $a-p^{2} \geq 0$ is true, then as we have already shown

$$
\begin{aligned}
g(u, v) & =\sqrt{a u^{2}+2 b u v+c v^{2}}+p u+q v+r \\
& \geq \sqrt{a u^{2}+2 b u v+c v^{2}}-\sqrt{p^{2} u^{2}+2 p q u v+q^{2} v^{2}}+r \\
& \geq r=g(\hat{u}, \hat{v}) .
\end{aligned}
$$

In the case $a-p^{2}<0$ we have shown for $s<0$ that $g(s, 0)<r=g(\hat{u}, \hat{v})$. Since each local extremum of a convex function is also a global extremum, therefore the pair $(\hat{u}, \hat{v})$ does not give the extremum of $g(u, v)$.

The considered cases can be resumed in the form (9)

Lemma 4. The function

$$
g(t)=\sqrt{a t^{2}+2 b t+c}+p t+q \quad\left(a>0, a c-b^{2}>0\right)
$$

is convex and for the problem $g(t) \longrightarrow$ min the following holds:

1. If $p \leq-\sqrt{a}$, then $g$ does not possess a local minimum and $g$ is decreasing.

2. If $p \geq \sqrt{a}$, then $g$ does not possess a local minimum and $g$ is increasing.

3. If $a-p^{2}>0$, then

$$
g_{\min }=g\left(t_{0}\right)=\frac{1}{2} \sqrt{\left(a c-b^{2}\right)\left(a-p^{2}\right)}-\frac{b p}{a}+q
$$

where

$$
t_{0}=-\frac{b}{a}-\frac{p}{a} \sqrt{\frac{a c-b^{2}}{a-p^{2}}} .
$$

Proof. Put for brevity $Q=Q(t)=a t^{2}+2 b t+c$ and $\Delta=a c-b^{2}$. Then

$$
g^{\prime}(t)=\frac{a t+b}{\sqrt{Q}}+p \quad \text { and } \quad g^{\prime \prime}(t)=\frac{\Delta}{\sqrt{Q^{3}}}>0
$$

The equation $g^{\prime}(t)=0$ has a solution only if $a-p^{2}>0$ and in this case the solution is given by (11). For $p \leq-\sqrt{a}$ we have $g^{\prime}(t)<0$ and for $p \geq \sqrt{a}$ we have $g^{\prime}(t)>0$ 
Remark 1. In the first two cases the function $g$ is bounded below if $p=-\sqrt{a}$ and $p=\sqrt{a}$, respectively.

As an obvious consequence of Lemma 4 we get the following

Lemma 5. Let the function $g=g(t)$ be defined by (10) and consider the problem $g(t) \longrightarrow \min \left(t_{1} \leq t \leq t_{2}\right)$. The solution $g_{\min }$ of this problem is the following:

1. If $p \leq-\sqrt{a}$, then $g_{\min }=g\left(t_{2}\right)$.

2. If $p \geq \sqrt{a}$, then $g_{\min }=g\left(t_{1}\right)$.

3. If $a-p^{2}>0$, then $g_{\min }= \begin{cases}g\left(t_{1}\right) & \text { if } t_{0}<t_{1}<t_{2} \\ g\left(t_{0}\right) & \text { if } t_{1} \leq t_{0} \leq t_{2} \\ g\left(t_{2}\right) & \text { if } t_{1}<t_{2}<t_{0}\end{cases}$

Here the number $t_{0}$ is defined by (11).

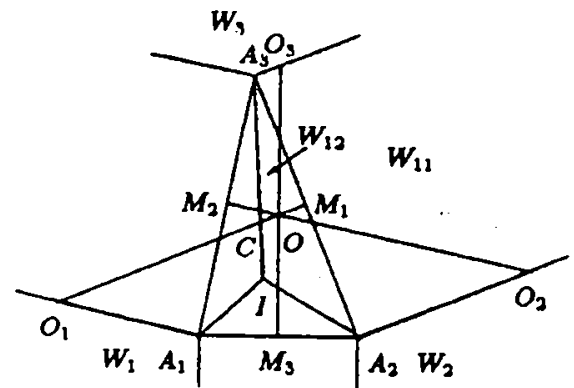

Figure 5

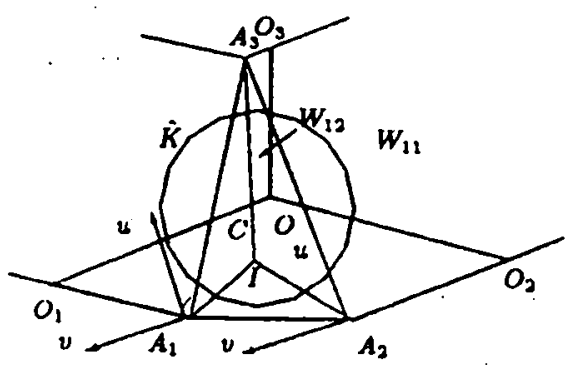

Figure 6

Now our main result follows.

Theorem 4. The center of the circle of the best Hausdorff approximation of the triangle $T$ is the point $C$ being the intersection of the midline of the longest side and the bisectrix against the shortest side.

Proof. We accept for determination that

$$
a_{1} \geq a_{2} \geq a_{3}
$$

Figure 5 gives an idea of the arising situation. Put for brevity

$$
g(X)=2 \hat{h}(X)= \begin{cases}r_{j}(X)-d_{i}(X) & \text { for } X \in W_{i j}(i, j=1,2,3) \\ r(X)+r_{i}(X) & \text { for } X \in W_{i}(i=1,2,3)\end{cases}
$$

where

$$
W_{i j}=\left\{X \in W \mid r(X)=r_{j}(X) \text { and } d(X)=d_{i}(X)\right\} .
$$

If $X$ is an interior point of $W_{i}$, then

$$
g(X)=r(X)+r_{i}(X)>r(X)>r\left(A_{i}\right)=g\left(A_{i}\right) .
$$


Therefore the minimum of $g$ is attained on $W$.

Next we show that $g_{\text {min }}$ is not attained in an interior point of $W_{i j}$. Let $X \in W_{11}$. We introduce an orthogonal coordinate system $A_{1} u v\left(A_{1} u \| A_{2} A_{3}\right)$ (see Figure 6). If $X(u, v)$ are the coordinates of $X$, then

$$
g=\sqrt{u^{2}+v^{2}}-v-h_{1}
$$

where $h_{1}$ is the length of the height through $A_{1}$. In this case $g$ is a smooth function on $W_{11}$. Applying Lemma 3 with $a=1, b=0, c=1$ and $p=0, q=-1, r=-h_{1}$ we see that the local minimum $g_{\min }=-h_{1}$ is attained for $(u, v)=(0,-t)$ with $t \leq 0$, i.e. on the positive $v$-semiaxe, however it has no common points with $W_{11}$. Similarly one proves that $g$ attains no local extrema in interior points of $W_{22}$ and $W_{33}$.

Let $X \in W_{12}$. Introduce an orthogonal coordinate system $A_{2} u v\left(A_{2} u\right.$ coincides with $A_{2} A_{3}$ ), shown on Figure 6. Then

$$
g=\sqrt{u^{2}+v^{2}}-v
$$

is a smooth function on $W_{12}$. Applying Lemma 3 we see that the local minimum $g_{\min }=0$ is attained for $(u, v)=(0,-t)$ with $t \leq 0$, i.e. on the positive $v$-axix. However it has no common points with $W_{12}$. In a similar way we show that $g$ attains no local extrema on the set $W_{i j}(i \neq j)$.

These reasonings show that $g$ attains its minimum on a point of the segments $O O_{i}$ and $A_{i} I(i=1,2,3), O C$ or $I C$ being parts of the midlines and the bisectrices. Here $O$ is the center of the circumscribed circumference, $I$ is the center of the inscribed circumference, $O_{i}$ are intersecting points of the midlines and perpendiculars of the sides through the vertices. We investigate further $g$ on each of the mentioned segments.

Case 1. $\min \left\{g(X) \mid X \in O_{2} O\right\}=g(O)$. Let $X \in O_{2} O \subset W_{11}$. We put $M_{2} X=t$, where $M_{2}$ is the middle of $A_{3} A_{1}$. Then $r_{1}=\sqrt{t^{2}+\frac{1}{4} a_{2}^{2}}, d_{1}=\frac{1}{2} a_{2} \sin A_{3}-t \cos A_{3}$, and

$$
g=r_{1}-d_{1}=\sqrt{t^{2}+\frac{1}{4} a_{2}^{2}}+t \cos A_{3}-\frac{1}{2} a_{2} \sin A_{3} \quad\left(t_{1} \leq t \leq t_{2}\right)
$$

where $t_{1}=\frac{1}{2} a_{2} \cot A_{2}$ is the value of $t$ corresponding to the point $O$ and $t_{2}=M_{2} O_{2}$. The assertion now follows from Lemma 5 with

$$
\begin{gathered}
a=1, \quad b=0, \quad c=\frac{a_{2}^{2}}{4}, \quad p=\cos A_{3}, \quad q=-\frac{1}{2} a_{2} \sin A_{3} \\
a c-b^{2}=\frac{a_{2}^{2}}{4}>0 ; \quad a-p^{2}=\sin ^{2} A_{3}, \quad t_{0}=-\frac{1}{2} a_{2} \cot A_{3}<t_{1} .
\end{gathered}
$$

Case 2. $\min \left\{g(X) \mid X \in O_{3} O\right\}=g(O)$. Let $X \in O_{3} O \subset W_{12}$. We put $M_{3} X=t$ where $M_{3}$ is the middle of $A_{1} A_{2}$. Then $r_{2}=\sqrt{t^{2}+\frac{1}{4} a_{3}^{2}}$ and

$$
g=r_{2}-d_{1}=\sqrt{t^{2}+\frac{1}{4} a_{3}^{2}}+t \cos A_{2}-\frac{1}{2} a_{3} \sin A_{2} \quad\left(t_{1} \leq t \leq t_{2}\right)
$$


where $t_{1}=\frac{1}{2} a_{3} \cot A_{3}$ is the value of $t$ corresponding to the point $O$ and $t_{2}=M_{3} O_{3}$. The assertion now follows from Lemma 5 with

$$
\begin{gathered}
a=1, \quad b=0, \quad c=\frac{a_{3}^{2}}{4} \quad p=-\cos A_{2}, \quad q=-\frac{1}{2} a_{3} \sin A_{2} \\
a c-b^{2}=\frac{a_{3}^{2}}{4}>0, \quad a-p^{2}=\sin ^{2} A_{2}, \quad t_{0}=-\frac{1}{2} a_{3} \cot A_{2}<t_{1} .
\end{gathered}
$$

Case 3. $\min \left\{g(X) \mid X \in O_{1} C\right\}=g(C)$. Let $X \in O_{1} C \subset W_{23}$. We put $M_{1} X=t$, where $M_{1}$ is the middle of $A_{2} A_{3}$. Then

$$
g=r_{3}-d_{2}=\sqrt{t^{2}+\frac{1}{4} a_{1}^{2}}+t \cdot \cos A_{3}-\frac{1}{2} a_{1} \sin A_{3} \quad\left(t_{1} \leq t \leq t_{2}\right)
$$

where $t_{1}=M_{1} C=\frac{1}{2} a_{1} \tan \frac{1}{2} A_{3}$ and $t_{2}=M_{1} O_{1}$. The assertion now follows from Lemma 5 with

$$
\begin{gathered}
a=1, \quad b=0, \quad c=\frac{a_{1}^{2}}{4}, \quad p=\cos A_{3}, \quad q=-\frac{1}{2} a_{1} \sin A_{3} \\
a c-b^{2}=\frac{a_{1}^{2}}{4}>0, \quad a-p^{2}=\sin ^{2} A_{3}, \quad t_{0}=-\frac{1}{2} a_{1} \cot A_{3}<t_{1} .
\end{gathered}
$$
Then

Case 4. $\min \{g(X) \mid X \in C O\}=g(C)$. Let $X \in C O \subset W_{12}$. We put $M_{1} X=t$.

$$
g=r_{2}-d_{1}=\sqrt{t^{2}+\frac{1}{4} a_{1}^{2}}-t \quad\left(t_{1} \leq t \leq t_{2}\right)
$$

where $t_{1}=M_{1} O=\frac{1}{2} a_{1} \tan \frac{1}{2} A_{3}$ and $t_{2}=M_{1} C=\frac{1}{2} a_{1} \tan \frac{1}{2} A_{3}$. The assertion now follows from Lemma 5 with

$$
\begin{gathered}
a=1, \quad b=0, \quad c=\frac{a_{1}^{2}}{4}, \quad p=-1, \quad q=0 \\
a c-b^{2}=\frac{a_{1}^{2}}{4}>0, \quad p=-\sqrt{a} .
\end{gathered}
$$

Case 5. $\min \left\{g(X) \mid X \in A_{1} I\right\}=g(I)$. Let $X \in A_{1} I \subset W_{33}$. We put $A_{1} X=t$. Using the cosine theorem for triangle $A_{3} A_{1} X$ we get

$$
g=r_{3}-d_{3}=\sqrt{t^{2}-2 t a_{2} \cos \frac{1}{2} A_{1}+a_{2}^{2}}-t \sin \frac{1}{2} A_{1} \quad\left(t_{1} \leq t \leq t_{2}\right)
$$

where $t_{1}=0$ and $t_{2}=A_{1} I=\left(p-a_{2}\right) / \cos \frac{1}{2} A_{1}$. The assertion now follows from Lemma 5 with

$$
\begin{gathered}
a=1, \quad b=-a_{2} \cos \frac{1}{2} A_{1}, \quad c=a_{2}^{2}, \quad p=-\sin \frac{1}{2} A_{1}, \quad q=0 \\
a c-b^{2}=a_{2}^{2} \sin ^{2} \frac{1}{2} A_{1}, \quad a-p^{2}=\cos ^{2} \frac{1}{2} A_{1} .
\end{gathered}
$$


Case 6. $\min \left\{g(X) \mid X \in A_{2} I\right\}=g(I)$. In this case the assertion can be derived similar to the Case 5.

Case 7. $\min \left\{g(X) \mid X \in A_{3} C\right\}=g(C)$. Also in this case the assertion can be derived similar to the Case 5 . Then

Case 8. $\min \{g(X) \mid X \in C I\}=g(C)$. Let $X \in C I \subset W_{23}$. We put $A_{3} X=t$.

$$
g=r_{3}-d_{2}=t-t \sin \frac{1}{2} A_{3}=t\left(1-\sin \frac{1}{2} A_{3}\right) \geq g(C) .
$$

Comparing all the Cases $1-8$ we draw the conclusion that min $g=g(C)$

Remark 2. If $\hat{K}=K(C, \hat{\rho})$ is the Hausdorff nearest circle for the triangle $T$ (see Figure 6) satisfying for determination (12) and $\hat{h}=h(T, \hat{K})$, then the proof of Theorem 4 gives

$$
\hat{\rho}=\frac{1}{4} a_{1} \frac{1+\sin \frac{1}{2} A_{3}}{\cos \frac{1}{2} A_{3}} \quad \text { and } \quad \hat{h}=\frac{1}{4} a_{1} \frac{1-\sin \frac{1}{2} A_{3}}{\cos \frac{1}{2} A_{3}} .
$$

\section{References}

[1] Alt, H. and H. Wagener: Approximation of polygons by rectangles and circles. Bull. EATCS 36 (1988), $103-113$.

[2] Arnold, R.: Zur $L^{2}$-besten Approximation eines konvexen Körpers durch einen bewegten konvexen Körper. Monatsh. Math. 108(1989), 277 - 293.

[3] Bani, M.S. and B.L. Chalmers: Best approximation in $L^{\infty}$ via iterative Hilbert space procedure. J. Approx. Theory 42 (1984), $173-180$.

[4] Brudnyj, Yu.A. and I.P. Irodova: Applied approximation theory (in Russian). Yaroslavl': Gosudarstvennyj Universitet 1986.

[5] Florian, A.: Approximation of convex discs by polygons. Discrete Comput. Geom. 1 (1986), $241-263$.

[6] Hettich, R. and P. Zenke: Numerische Methoden der Approximation und semi-infiniten Optimierung. Stuttgart: B.G. Teubner 1982.

[7] Karlin, S. and W.J. Studden: Tchebycheff systems: With application in analysis and statistics. New York - London - Sydney: Wiley \& Sons Intersci. Publ. 1966.

[8] Kenderov, P.S.: Approximation of plane convex compacta by polygons. In: Constructive Function Theory. Proc. Int. Conf. Varna. Sofia: Bulgarian Acad. Sci. 1981, pp. 376 381.

[9] Laurent, J.-P.: Approximation et optimization. Paris: Hermann 1972.

[10] LeichtweiB, K.: Konvexe Mengen. Berlin: Dt. Verlag Wiss. 1980.

[11] Lessak, M.: Approximation of plane convex bodies by centrally symmetric bodies. J. Lond. Math. Soc. (Serie II) 40 (1988), 369 - 377. 